TRANS · núm. $20 \cdot 2016$

ARTICULO $\cdot$ 41-55
Este estudio sitúa en su contexto cultural, histórico y político la génesis de las dos traducciones, la primera al catalán y la primera al español, en el siglo XX, de la novela La Princesse de Clèves (1678), un clásico francés próximo a la préciosité, obra de Madame de La Fayette. El texto catalán (1923) coincide con el movimiento noucentista, impulsor, en el marco de la Mancomunitat de Catalunya, de reformas literarias y lingüísticas, especialmente en el ámbito de la traducción de literatura universal. La traducción al español (1924) es el resultado del interés por crear una industria editorial -Calpe, El Sol, La Papelera Española- que pudiera difundir una corriente ideológica laicista, al tiempo que ofreciese, en el ámbito literario, una selección universal del pensamiento y la estética. El estudio ofrece asimismo un análisis comparativo de las dos traducciones y una exploración sobre los dos traductores de una obra considerada como precursora de la novela moderna francesa.

PALABRAS ClAVE: Traducción literaria; Madame de La Fayette; Princesse de Clèves; Rafael Marquina; J. Sesplugues; novela moderna

\title{
Las primeras traducciones al catalán y al español de La Princesse de Clèves (I678). Un clásico francés que abre paso a la novela moderna ${ }^{\mathrm{I}}$
}

\author{
Eusebi Coromina Pou \\ Universitat de Vic (Barcelona)
}

I Este artículo se inscribe en el Grupo de Estudios de Género: Traducción, Literatura, Historia y Comunicación (GETLIHC) (20I4, SGR 62), reconocido por la Agencia de Gestión y Ayudas Universitarias de la Generalitat de Catalunya, y en el proyecto «Traducción y censura: género e ideología (1939-2000)», con el número de referencia FFI2OI4-52989- $\mathrm{C}_{2-2}-\mathrm{P}$, financiado por el Ministerio de Economía y Competitividad.
The First Translations into Catalan and Spanish of La Princesse de Clèves (1678): A French classic ushering in the modern novel

This study examines the cultural, historical and political context of the genesis of the two twentieth-century translations - the first into Catalan and the first into Spanish - of the classic seventeenth-century work of French préciosité by Madame de La Fayette. The Catalan text (1923) coincides with the Noucentista movement, which in the framework of the Commonwealth of Catalonia fostered innovation in literature and language, particularly in the translation of worldwide literature into Catalan. The Spanish translation (1924) stems from interest in creating a publishing industry - Calpe, El Sol, La Papelera Española - capable of disseminating a secular ideological current, while at the same time offering a wide-ranging selection of thought and aesthetics in literature. Further, this study includes a comparative analysis of the two translations and explores the two translators of a work regarded as the harbinger of the modern French novel.

KEY WORDS: Literary translation; Madame de La Fayette; Princesse de Clèves; Rafael Marquina; J. Sesplugues; modern novel 


\section{EL DETONANTE DE LA VERSIÓN} CATALANA

42
La Princesse de Clèves (I678) de Madame de La Fayette (I634-I693), un clásico del XVII con trazas del preciosismo (préciosité) francés, contribuye a dar paso a la novela moderna francesa, al tiempo que refuerza la intervención de la mujer en la literatura de la época, ya sea como autora y como protagonista de una historia literaria ${ }^{2}$.

No será hasta 245 años más tarde cuando se traducirá al catalán (I923) 3 y después al español $(\mathrm{I} 924)^{4}$, ya en el siglo xx, gracias tanto a Rafael Marquina como a J. Sesplugues (pseudónimo que utiliza el traductor de la versión en español). A la primera traducción catalana le sigue solamente otra, en $1990^{5}$; a la primera traducción en español, le siguen otras ocho (en i8 ediciones distintas $)^{6}$.

La primera versión catalana aparece en la Biblioteca Literària de Editorial Catalana, creada como sociedad anónima en Barcelona en I9I7 e instalada en la misma sede de la redacción del periódico La Veu de Catalunya, portavoz de la Lliga Regionalista. Editorial Catalana, que publicará cinco periódicos más, entre ellos el mensual artístico D'Ací $i$ d'Allà (1918-1936), en

2 Para la versión original en francés se ha optado por la edición de La Fayette (200o), de Joël Surcouf, de l'Association des Bibliophiles Universels (ABU), con sede en París.

3 La versión en catalán de Rafael Marquina consultada corresponde a La Fayette (1923).

4 La versión en español de J. Sesplugues consultada corresponde a La Fayette (1924).

5 A cargo de Josep A. Grimalt (Barcelona: Proa, I990).

6 Debidas a Federico Carlos Sainz de Robles (Barcelona: Apolo, 194I), Antonio G. Linares (Madrid: Aguilar, I96I), José M ${ }^{\text {a }}$ Claramunda Bes (Barcelona: Zeus, I964), J. Pérez de Hoyo (Madrid: Clásicos Universales, I970), Vicente Clavel (Estella: Salvat, I97I), Emma Calatayud (Barcelona: Bruguera, I982), Ricardo Permanyer (Barcelona: Planeta, I983) y Ana María Holzbacher (Madrid: Cátedra, 1987). el catálogo de la Biblioteca Literària pone de manifiesto el objetivo principal de la colección:

Era un afany universalment sentit a tot Catalunya el que s'incorporessin a la nostra llengua, per fer-les assequibles a tothom, les obres mestres de totes les literatures. [...] Doncs això és el que principalment aquesta Biblioteca es proposa, i a aquest objecte hem encarregat als nostres millors escriptors la traducció directa, des de la llengua originària a la nostra, de totes les obres de més anomenada; i podem garantir que constituiran un monument literari d'actualitat perenne i el mitjà d'educació per a les nostres generacions. ${ }^{7}$

\section{I.I. El Noucentisme}

La fundación de Editorial Catalana forma parte de los objetivos del Noucentisme cultural y político del primer tercio del siglo $\mathrm{xx}$ (Coromina, 20I2). Partiendo de una idea del político Enric Prat de la Riba, primer presidente de la Mancomunitat de Catalunya (I914-1925), con la nueva editorial se pretende extender la influencia social del partido conservador Lliga Regionalista, al que pertenece, al tiempo que constituye un intento de ampliar el mercado lector entre la clientela política y la población en general. El Noucentisme más distintivamente cultural tenía por norte la consecución de un catalán literario común, moderno y flexible, útil especialmente para usos formales y elevados. Si Pompeu Fabra, junto a la Secció Filològica del

7 Texto aparecido en el catálogo inicial y promocional, publicado probablemente en I9I8 (Llanas, 2005: 296). Traducción propia: «Era un afán sentido universalmente en toda Cataluña el que se incorporaran a nuestra lengua las obras maestras de todas las literaturas, a fin de hacerlas asequibles a todo el mundo. [...] Por consiguiente, eso es lo que se propone la Biblioteca, y a ese fin hemos encargado a nuestros mejores escritores la traducción directa de las obras de más renombre, desde la lengua originaria a la catalana; y podemos garantizar que constituirán un monumento literario de actualidad perenne y un medio de educación para nuestras generaciones.» 
Institut d'Estudis Catalans, trabajaba ya en la codificación lingüística, era necesario también un canon literario al que aplicar el nuevo código idiomático, interferidos ambos por numerosas formas y construcciones del español. A este fin se consideró útil promover la traducción de literatura universal a fin de obtener, a la luz de la práctica traductora, un modelo literario y lingüístico propios, maleables y sin interferencias (Malé, 2007). Simultáneamente, las traducciones podían ayudar a superar el localismo, de acuerdo con los noucentistes, acercando la identidad lingüístico-cultural propia a la cultura universal.

El poeta y traductor Josep Carner es el primer director literario (I9I7-I923) de Editorial Catalana. Prat de la Riba lo había incorporado ya en la Secció Filològica de I'IEc, no en calidad de lingüista, como Fabra, sino en calidad de escritor. De la colección Biblioteca Literaria (II8 títulos entre I9I8 y I93I) Carner traducirá I6 obras junto a otros traductores/escritores: Carles Riba, Llorenç Riber, Joaquim Ruyra, Rodolf J. Slaby, Marià Manent, M. Antònia Salvà, Emili Vallès, Tomàs Garcés, Carles Soldevila o Rafel Marquina. Destacan las versiones catalanas de Dickens, Twain, Andersen, E. A. Poe, Shakespeare, Goethe, Elliot, Molière, Kipling, La Fontaine, Walter-Scott, Mme. de La Fayette, Manzoni, Daudet, Pushkin, Conrad o Maeterlinck.

\section{I.2. El traductor Rafael Marquina}

Es en el contexto noucentista cuando aparece la primera traducción peninsular del siglo $\mathrm{XX}$ de La princesa de Clèves (1923), obra de Rafael Marquina y Angulo ${ }^{8}$ (Barcelona, I887 - la Habana, I960). Con 36 años, Marquina es un

8 En Portugal aparece en 1962 gracias a João Cabral do Nascimento (A princesa de Clèves. Lisboa: Estúdios Cor). noucentista de pies a cabeza, cultural y políticamente hablando. ${ }^{9}$ Llega a secretario de Francesc Cambó en el período en que este ejerce por dos veces como ministro del gobierno de Antonio Maura (I918-1922). Durante su estancia en Madrid como secretario de Cambó, Rafael Marquina y Mercè Audouard tuvieron, en r921, a su segundo hijo, Rafael Marquina Audouard, que sería arquitecto y diseñador. Mercè Audouard era la hija mayor del reconocido fotógrafo y retratista Pau Audouard, de origen francés (la Habana I856 - Barcelona I9I9), fotógrafo oficial de la Exposició Universal de Barcelona de 1888. Fue, pues, suegro de Rafael Marquina Angulo, quien lo aficionó al teatro hasta el punto de que Pau Audouard hizo una corta carrera como dramaturgo diletante, bajo el pseudónimo de Joan Aler.Los estudios de filosofía y letras de Rafael Marquina, su formación como crítico teatral y la circunstancia de ser hermano de Eduardo Marquina, hombre de teatro a quien admiraba profundamente, lo conducen necesariamente a las artes escénicas. Dirige la revista Teatràlia (entre 1908 y 1909) y es portavoz de los jóvenes noucentistes, al tiempo que estrena distintas piezas propias (Serra i Casals, 1996).

No es de extrañar la aparición de La Princesa de Clèves en el proyecto de Editorial Catalana. Madame de La Fayette da a la literatura francesa una gran obra que se inscribe en el clasicismo $\mathrm{y}$, en parte, en el preciosismo (la preciositê), coincidentes en cierta medida con los anhelos noucentistes de perfección, de control de la razón, de orden, equilibrio, deseo de universalidad y perdurabilidad (Sgard, 2000). El preciosismo, corriente estética de afirmación aristócrata, lleva consigo la marca de la voluntad de elegancia y refinamiento en el ámbito de las formas, del

9 Redactor de La Veu de Catalunya, publica en 1925 una breve biografía del político de la Lliga Francesc Cambó. 
gusto, de las costumbres y también del idioma, al tiempo que incorpora a la mujer en los cenáculos de intelectuales y artistas (Timmermans, 1993).

\section{MADAME DE LA FAYETTE}

Madame de La Fayette, de nombre de pila Marie-Madeleine, nace en París en I634 en el entorno familiar del cardenal Richelieu. Su padre, Marc Pioche de la Vergne, albéitar del rey y lugarteniente en el Gobierno, muere en I649 en Le Havre, donde reside la familia, quedando huérfana Marie-Madeleine a los quince años. Es en el mismo I649 cuando Mlle. de Scudéry i Descartes publican, respectivamente, Le Grand Cyrus y Traité des Passions. Marie-Madeleine, un año más tarde, es nombrada dama de honor de la reina Ana de Austria y se relaciona con el gramático Gilles Ménage, que le enseña latín e italiano, y la introducirá en los salones literarios preciosistas de la época: un savant vaniteux et fort empressé auprès des femmes, plus âgé quèelle de vingt ans [...] qui lui communique une partie de son vaste savoir. (Pingaud, I978: 25). En I650, su madre, Isabelle Pena, se casa con Renaud de Sévigné, tío de Marie de Sévigné, reconocida per el cultivo del género epistolar, con quien Madame de La Fayette mantendrá una fuerte amistad (Pingaud, 1978), especialmente a partir de 1657.

Los preciosistas, reunidos en los salones literarios de París, discuten especialmente de poesía, cuyo centro de interés es el amor, tratado casi siempre como pura inclinación del espíritu (Lathuillère, I966). Son especialmente mujeres quienes contribuyen a crear este movimiento refinado y elegante que es el preciosismo, partidario además de la pureza y riqueza del lenguaje, por lo que promoverá en 1694 la primera edición del Dictionnaire de l'Académie Française. En
I647 ya habían sido publicadas las Remarques sur la langue françoise: utiles à ceux qui veulent bien parler et bien escrire, de Vaugelas, ${ }^{\mathrm{IO}}$ cuyas reglas se inspiraban en el habla de la corte ${ }^{\mathrm{II}}$ (Lathui1lère, I966). Esa renovación lingüística preconizada por los preciosistas en literatura tiene su paralelismo con la reforma y modernización del catalán literario promovidas por el Noucentisme a través de Pompeu Fabra y el Institut d'Estudis Catalans a principios del siglo XX.

Marie-Madeleine, lectora de Mlle. de Scudéry -consta que lee Le Grand Cyrus en 1654-, frecuentaba su salón y también el de la marquesa de Rambouillet. Un año más tarde se casa con François, conde de La Fayette, viudo de 38 años, a quien acompaña durante un tiempo por sus tierras de Auvergne y Bourbonnais, encargándose de la administración de sus fincas. Vuelve a París definitivamente en 1659 después de haber dado a luz a dos hijos: Louis (I658-I729), que será abad, y René Armand (I659-I694), soldado. Frecuenta el salón de Mme. Du Plessis-Guénégaud, y publica en 1662 su primera obra, también sin nombre de autor, La princesse de Montpensier, por el impresor y editor parisino Claude Barbin. A partir de 1665 estrecha su amistad con el duque-escritor La Rochefoucauld, asiduo de los salones preciosistas, quien seguirá de cerca el proceso de escritura de La Princesse de Clèves, que empieza en 1672, seis años antes de su publicación. Parece ser que La Rochefoucauld habría participado en la elaboración de La Princesse de Clèves, sin que por ello fuera su autor, como se ha habría dicho (Malandain, 1989: 27).

ro Vaugelas define el buen uso como «la façon de parler de la plus saine partie de la Cour, conformément à la façon d'escrire de la plus saine partie des Autheurs du temps». (Chaurand, 1999: 237).

II Se debe a los preciosistas el proyecto de simplificar la ortografia. Utilizan ciertas formas que serán admitidas por la Académie française, como auteur por auther, être por estre, aîné por aisné; répondre por respondre. 


\section{I. La Princesse de Clèves: novela moderna, clásica y preciosista}

La Princesse de Clèves aparece sin nombre de autor en i678, publicada también por Claude Barbin. En el siglo XviI ser autor literario no era precisamente bien visto en la buena sociedad. Declararse autor de una obra podía ser considerado, además, un acto de vanidad, como resalta Rapin (1677: 98). No obstante, La Fayette recibió consejos e influencias de distintos autores para la elaboración de la novela: los mencionados La Rochefoucauld y Mme. de Sévingé, además del poeta, narrador y traductor Segrais o del teórico de la novela Pierre-Daniel Huet. Hay unanimidad en considerar a La Fayette como autora de la Princesse, o al menos de su arquitectura fundamental. El libro, no obstante, no aparecerá con el nombre de su autora hasta I780, cien años después de la primera edición.

\section{I.I. Precursora de la novela moderna}

La Princesse de Clèves es una narración clave en la historia de la literatura francesa. Es considerada una de las primeras novelas modernas, que rompe con la tradición del xvII (Lever, I98I), situando en primer plano el análisis psicológico, la vida interior de los personajes. Huye de la novela río (roman-fleuve) de principios del XVII, que relata historias inverosímiles con múltiples acciones novelescas exteriores a lo largo de numerosas páginas (el caso extremo es Le Grand Cyrus, 1649-1653, de Madeleine de Scudéry, que cuenta con 13.095 páginas). La historia de $L a$ Princesse de Clèves, en cambio, se desarrolla a lo largo de 200 páginas de promedio entre las distintas ediciones, y centra su acción alrededor de un personaje, anclada en la verosimilitud, ya sea histórica (la Corte de los Valois bajo el reinado de Enrique II), ya sea psicológica (los personajes evolucionan conforme a la evolución natural de los sentimientos). Se trata, también, de una novela clásica en el sentido de que respeta a su manera los principios de unidad de tiempo (la historia se desarrolla en el período de un año), 45 unidad de acción (se centra alrededor de una sola acción) y verosimilitud (histórica y psicológica). Pero La Princesse de Clèves es en parte también una novela preciosista, especialmente por su concepción del amor, próxima a la de la préciosité: un amor puro, virtuoso, respetuoso con las convenciones de la época (Pommier, I990). Es, además, testimonio del importante rol desarrollado por las mujeres en la literatura y en la vida cultural del xviI, marcado por la corriente de la préciosité, y representado en este caso por la autora de la obra y por el protagonismo femenino de la historia.

\section{I.2. La Princesse de Clèves: Historia de una renuncia}

Como ya hemos señalado en otro estudio (Coromina, 20I2), la autora sitúa la historia, a mediados del siglo XVI (un siglo antes de la publicación de la obra), en la corte del rey Enrique II, en el Palacio del Louvre de París, donde deambulan numerosos personajes históricos. Mademoiselle de Chartres, una joven huérfana de 16 años, de gran belleza e inexperta, recién llegada a la corte, se convierte en princesa al casarse con el Príncipe de Clèves, de quien no está verdaderamente enamorada. La princesa entabla entonces una verdadera lucha interior entre la fidelidad a su marido y la verdadera pasión que siente por el joven duque de Nemours. Ambos se profesan de forma no declarada una atracción mutua, próxima al ideal del amor puro, pero motivo de sufrimiento para ella como consecuencia del combate que sostiene al intentar huir, por la educación recibida de su madre, del amor indebido y perturbador hacia Nemours. 
La protagonista termina confesando a su marido, como prueba de fidelidad, la atracción que siente por otro hombre sin nombrarlo, $y$ 46 le pide que pueda abandonar la corte para no caer así en la tentación. El duque, a escondidas, escucha sorprendido la confesión (l'aveu), ya que se había acercado al pabellón del parque donde tiene lugar la declaración para espiar a la princesa. El príncipe de Clèves, devorado por los celos, intenta descubrir el nombre del amante y manda seguir al duque de Nemours, de quien sospecha ser el amante de su esposa. No pudiéndolo demostrar, el marido cae enfermo y muere, lo que la princesa interpreta como obstáculos infranqueables para llevar a cabo su pasión, ahora ya legítima. Decide renunciar al duque a pesar de las pruebas de estimación que éste le manifiesta. En el transcurso de un último encuentro, la protagonista le comunica su renuncia definitiva apelando al deber al marido y a la tranquilidad de conciencia. La princesa se retirará primero en terres quelle avait vers les Pyrénées; luego, en una casa de religiosas, y finalmente en la suya propia, et sa vie, qui fut assez courte, laissa des exemples de vertu inimitables (La Fayette, 2000: 8I).

\section{FIDELIDAD AL ORIGINAL}

Los dos traductores de la novela, Rafael Marquina y J. Sesplugues, siguen fielmente el original francés en lo que concierne al sentido, al tono y al estilo de un clásico del XVII francés. $\mathrm{El}$ original parece especialmente indicado para un traductor noucentista en la búsqueda de un lenguaje formal y elevado.

El original de Madame de La Fayette prescinde, como no podía ser de otra forma, de la variación de registros lingüísticos: narrador y discurso de los personajes utilizan un mismo lenguaje refinado y artificioso, supuestamente propio de un medio aristócrata y cortesano del

siglo XVI, sin distinción de funciones o de niveles de relación. Esta reducción de los registros a un solo estilo formal y ornamental coincide en la versión catalana con los postulados y con la práctica del Noucentisme (Pericay y Toutain, 1996). Esa unificación está plenamente justificada, así como el trato de vos que se dispensan los personajes en ambas traducciones. No sucede de este modo en distintas comedias del coetáneo Molière (1622-1673), en las que recurre a menudo al registro coloquial, como en Tartuffe ou l'imposteur, estrenada en 1664; L'Avare, representada por primera vez en I668, o Le Malade imaginaire, estrenada en 1673.

Ambas traducciones de La princesse de Clèves conservan los superlativos e hipérboles preciosistas (Coromina, 2012: I6I-I62) al calificar los sentimientos o acciones de los personajes, como muestra este fragmento (en negrita, Tabla I):

Las dos versiones recogen también las lítotes que presenta el original francés (Coromina, 20I2: I62). Conservan así el estilo preciosista y academicista del original, así como la tendencia a las secuencias sintácticas largas, con subordinación y coordinación, ya sea en el discurso de los personajes, ya sea en el relato del narrador, como muestra el siguiente fragmento, formado por una sola secuencia sintáctica (Tabla 2):

\section{EL DETONANTE DE LA VERSIÓN EN ESPAÑOL}

La traducción al español de J. Sesplugues aparece, como ya se ha apuntado, en 1924, en editorial Calpe, de Madrid, en la Colección Universal. J. Sesplugues es un pseudónimo de reminiscencias catalanas: existen el topónimo y el antropónimo catalanes Esplugues. Nos vamos a referir más adelante a la posible identidad del traductor.

Calpe (Compañía Anónima de Librería, Publicaciones y Ediciones), fundada en r918 
TABLA I.

EXPRESIONES HIPERBÓLICAS

FRANCÉS

Jamais cour n'a eu tant de belles personnes et d'hommes admirablement bien faits; et il semblait que la nature eût pris plaisir à placer ce qu'elle donne de plus beau, dans les plus grandes princesses et dans les plus grands princes. Madame Élisabeth de France, qui fut depuis reine d'Espagne, commençait à faire paraître un esprit surprenant et cette incomparable beauté qui lui a été si funeste.

[La Fayette, 2000: 4]
CATALÁN

ESPAÑOL

Mai cap altra [cort] ha tingut dones tan belles ni homes tan ben plantats; semblava que la Natura hagués tingut complaença a prodigar ço que ella dóna de més plaent, en les persones dels grans prínceps i de les altes princeses. Isabel de França, que fou després reina d'Espanya, començava a alliberar el seu talent sorprenent i aquella formosor seva que li ha estat tan funesta.

[Marquina. La Fayette, I923:8]
Jamás Corte alguna reunió tantas y tan hermosas damas ni hombres tan gallardos y gentiles. Parecía que la naturaleza se había complacido en distribuir sus más preciados dones entre las grandes princesas y los grandes príncipes. Isabel de Francia, que luego fué reina de España, empezaba a manifestar su ingenio sorprendente y aquella incomparable belleza que tan funesta la ha sido. [Sesplugues. La Fayette, I924:Io]

TABLA II.

SECUENCIAS LARGAS Y COMPLEJAS

FRANCÉS

Quand elle pensait encore que monsieur de Nemours voyait bien qu'elle connaissait son amour, qu'il voyait bien aussi que malgré cette connaissance elle ne l'en traitait pas plus mal en présence même de son mari, qu'au contraire elle ne l'avait jamais regardé si favorablement, qu'elle était cause que monsieur de Clèves l'avait envoyé quérir, et qu'ils venaient de passer une près-dînée ensemble en particulier, elle trouvait qu'elle était d'intelligence avec monsieur de Nemours, qu'elle trompait le mari du monde qui méritait le moins d'être trompé, et elle était honteuse de paraître si peu digne d'estime aux yeux même de son amant.

[La Fayette, 2000: 47]

\section{CATALÁN}

Demés, en pensar que el duc

de Nemours comprenia que era assabentada de la seva amor i que, malgrat això, no el tractava pas malament ni tan sols a presència del seu marit i que, al contrari, mai no li havia palesat tanta favor; que ella era causa que el príncep l'hagués enviat a cercar i que havien passat una tarda junts, cregué que era en intel-ligència amb el duc de Nemours, que enganyava el marit més digne de no ésser-ho i àdhuc tingué basarda de desmerèixer als ulls del seu amant.

[Marquina. La Fayette, I923: III]
Cuando pensaba que monsieur de Nemours sabía muy bien que ella conocía su amor, y que a pesar de esto no le trataba mal a la misma presencia de su marido, sino que antes al contrario lo trataba más cariñosamente que nunca; que era la causa de que su marido le hubiese enviado a buscar, y que acababan de pasar toda una tarde reunidos en particular, entonces juzgaba que esto significaba una avenencia con monsieur de Nemours, que engañaba a su marido, que era el que menos lo merecía de todos los maridos del mundo, y hasta llegaba a sentir vergüenza de parecer poco digna de estimación a los ojos mismos de su amante.

[Sesplugues. La Fayette, I924:I42-I43] 
por el ingeniero de origen vasco Nicolás María de Urgoiti (un año después de la fundación de Editorial Catalana), destaca por su aportación a la industria del sector editorial, y contribuye notablemente al desarrollo cultural en el primer tercio del siglo XX. La editorial desarrolla su actividad, especialmente en literatura y ciencia, entre I9I8 y I925, año en que se fusiona con la catalana Espasa.

\section{I. Proyecto comercial e intelectual}

Urgoiti había creado a principios de siglo La Papelera Española. Con la puesta en marcha de la editorial y la fundación del periódico $E l$ Sol en 1917, asegura el consumo de papel. De este modo, cuenta con una editorial y con un periódico, lo que garantiza la facturación de La Papelera Española (Cabrera, I994). Asimismo, cuenta con la Casa del Libro, la más importante librería de España de la época, abierta en la Gran Vía de Madrid en I923.

$\mathrm{El}$ proyecto tiene, sin embargo, un objetivo intelectual manifiesto, expresado en el primer editorial del diario $E l$ Sol, dirigido por Félix Lorenzo: «La renovación nacional en todas las esferas de la ciudadanía, de la cultura y del trabajo es la guía y enseña de nuestro pensamiento y nuestra voluntad.» («Editorial», I9I7) Urgoiti busca en el periódico y en Calpe el soporte para difundir una corriente laicista y progresista, por lo que cuenta con José Ortega y Gasset, Manuel García Morente o Ramón Menéndez Pidal, que serán responsables de distintas colecciones de la editorial.

\subsection{La Colección Universal}

Calpe, en este contexto, pone en marcha en I9I9 la Colección Universal. Es la primera colección de bolsillo de España y de las primeras del continente, diseñada siguiendo el modelo francés de la Bibliothèque Universelle (París) y el modelo alemán de la Universal Bibliothek (Leipzig). Para el profesor Manuel García Morente, responsable de la colección (Sánchez Vigil, 2006: 265):

La Compañía editora creyó desde el primer instante que era una obligación [...] dotar al público que habla español de una colección amplísima, donde tuviesen cabida las obras pretéritas que el tiempo hubiese consagrado [...], poner al alcance de todos ese tesoro espiritual de la humanidad.

Antonio Maura, en calidad de presidente de la Real Academia de Jurisprudencia y Legislación, señalaba también en el catálogo de la colección (Sánchez Vigil, 2006: 265):

Entiendo que la labor de la Colección Universal no puede ser más loable, porque el libro barato y selecto -sobre todo este último- no solo es propulsor de la general cultura, sino manantial inestimable de goce estético.

A su vez, Azorín opinaba lo siguiente sobre la colección: «Un enjambre de libritos doctos y amenos; un abejar de universalidad y humanidad: lo más exquisito y lo más popular del pensamiento humano.» (Sánchez Vigil, 2005:I23).

La Universal se interesa por difundir textos clásicos de origen diverso. Publica un promedio de 20 números mensuales, llegando al 980 en diciembre de 1924. Las obras presentan una media de roo páginas por ejemplar al precio de 50 céntimos cada volumen simple. Algunos títulos comprenden, según la extensión de la obra, más de un número: es el caso de La princesa de Clèves, que abarca del 974 al 976 (un total de 240 páginas). Incluye, entre otros autores, a la mencionada Madame de La Fayette, Daudet, Beaumarchais, La Rochefoucauld, Flaubert, Diderot, Molière, Dickens, Wilde, Shakespeare, Scott, Eliot, Stevenson, Leibniz, Hoffmann, Kant, Dante, Alfieri, Fogazzaro, Azeglio, Afanasiev, Chéjov, Andreiev, etc. De entre los tra- 
ductores destacan José Ortega y Gasset, Manuel Azaña, Cipriano Rivas Cheriff, Luis Astrana Marín o Pedro Salinas. Como veremos, también difunde traducciones de la literatura catalana y, evidentemente, originales de clásicos de la literatura española, como Alarcón, Cervantes, Quevedo o Rojas.

\section{LA AUTORÍA DE LA VERSIÓN EN ESPAÑOL}

La firma J. Sesplugues, como autor de la traducción de La Princesa de Clèves, es sin duda un pseudónimo. No hemos encontrado escritor y/o traductor alguno de una cierta relevancia o actividad que, con esa letra inicial y apellido, pudiera haber traducido La princesa de Clèves. Sin embargo, Givanel (1934) nos informa de la existencia del pseudónimo J. Sesplugues [Josep Sesplugues], que correspondería -dice- a Joan Pinós Marsell (nacido en r9or), de quien solo tenemos noticia de la publicación, como coautor, de Diners: drama en tres actes (1927). Sería hermano del prestigioso médico Tomàs Àngel Pinós Marsell (nacido en I892 en Vallfogona de Balaguer, Lleida), quien ejerció la profesión de médico en Balaguer y en Barcelona, y amplió estudios en Francia, Alemania, Gran Bretaña, Estados Unidos y Canadá. Pudiera ser, asimismo, que Joan Pinós fuera el firmante, con el pseudónimo J. Sesplugues, del artículo aparecido en la revista quincenal Segarra (I5 de julio de I926), periódico de arte y cultura, y portavoz de los intereses de la comarca (la Segarra) con motivo de un número extraordinario de homenaje a «la memoria del gloriós poeta català Mn. Jacinto Verdaguer» en el vigésimo quinto aniversario de su muerte. La colaboración de Joan Pinós (Sesplugues, 1926: I7-I8), breve, lleva por título «Verdaguer i la seva poesia», y está fechada en Manresa el día I4 de mayo de 1926. Nos dio la pista del citado artículo la noticia titulada «Un extraordinari de "Segarra” (1926), aparecida en el periódico La Veu de Catalunya.

Descartada la pista de Joan Pinós Marsell, nos asalta otra duda: la de que el traductor pudiera ser el mismo Rafael Marquina. Marquina utilizó pseudónimos en distintas ocasiones en su actividad periodística, como Farfarello o Un Ramblista. A su hijo, el arquitecto y diseñador Rafael Marquina Audouard, fallecido en junio de 2013, no le consta que su padre usase el pseudónimo J. Sesplugues, según nos había manifestado en diciembre de 20I2. En la misma conversación que mantuvimos manifestó sus dudas de que la versión catalana fuera debida únicamente a su padre, Rafael Marquina. Como ya se ha indicado, su esposa, Mercè Audouard, de origen francés, pudo haber colaborado directamente en dicha versión.

La hipótesis según la cual tras ese pseudónimo pudiera esconderse Rafael Marquina vendría avalada por el hecho de que la traducción al español aparece solo un año después de que se publique la catalana. La versión de J. Sesplugues aparece, por otro lado, en la Colección Universal, en la que Marquina ya había publicado distintas traducciones, esta vez del catalán al español. Conocía, por consiguiente, los entresijos de la editorial: había traducido para la Colección Universal obras de Víctor Català, Raimon Casellas, Eugeni d'Ors, Alfons Maseras, Curial e Güelfa (anònimo del XV), y ya en la posguerra Espasa-Calpe le publica, en 1946, otra obra d'Eugeni d'Ors. Marquina da a conocer a través de otras editoriales algunas obras más traducidas al español, como las de Àngel Guimerà, Apel·les Mestres, Josep M. de Sagarra o Santiago Rusiñol, hecho muy poco frecuente en la historia de las relaciones entre la literatura catalana y la española (Bacardí, 2007). Es significativa la traducción que hizo, en I9II, al español de La ben plantada [La bien plantada], 
de Eugeni d'Ors, que contiene el ideario estético del Noucentisme: la exaltación de la cultura y del clasicismo frente al Modernismo, a la naturaleza salvaje, a la inspiración espontánea y descontrolada. En el período comprendido entre I920 y 1925 lleva a cabo una gran actividad traductora: llega a traducir ocho obras, incluyendo la versión catalana de La princesa de Clèves.

\section{TRADUCCIONES Y TRADUCTORES DISTINTOS}

Hemos cotejado distintos fragmentos de la versión catalana y española en los cuatro capí-

tulos de ambas traducciones a fin de apreciar el grado de fidelidad entre ellas. De acuerdo con los resultados obtenidos, podemos concluir que el texto en español tiene, por así decirlo, personalidad propia, no está supeditado a la versión catalana, ya sea en el orden de las palabras o sintagmas y oraciones, ya sea en estructuras sintácticas, ya sea en categorías gramaticales o en la elección de formas léxicas. He aquí un resumen de ello (Tablas 3-6).

En efecto, la versión de J. Sesplugues se ha realizado partiendo directamente de un original francés y sin influencia de la traducción catalana. Probablemente la versión en español se

TABLA III.

ORDEN DE LOS SINTAGMAS Y LAS ORACIONES

\begin{tabular}{|c|c|c|}
\hline FRANCÉS & CATALÁN & ESPAÑOL \\
\hline $\begin{array}{l}\text { Le chevalier de Guise lui parais- } \\
\text { sait le plus redoutable par sa nais- } \\
\text { sance, par son mérite, et par l'éclat } \\
\text { que la faveur donnait à sa maison. } \\
\text { Ce prince était devenu amou- } \\
\text { reux de mademoiselle de Chartres } \\
\text { le premier jour qu'il l'avait vue. } \\
\text { [La Fayette, 2000: 8] }\end{array}$ & $\begin{array}{l}\text { Entre tots, li semblava més peri- } \\
\text { llós el cavaller de Guisa, pel seu } \\
\text { llinatge, pel seu mèrit i per la } \\
\text { brillantor que la privança donava } \\
\text { a la seva casa. Aquest príncep } \\
\text { s'enamorà de la senyoreta de } \\
\text { Chartes tan bon punt la veié. } \\
\text { [Marquina. La Fayette, I923: 20] }\end{array}$ & $\begin{array}{l}\text { El caballero de Guisa, que se } \\
\text { enamoró de la señorita de Char- } \\
\text { tres el primer día que la vio, le } \\
\text { parecía el más temible de todos, } \\
\text { tanto por su nacimiento y cua- } \\
\text { lidades personales como por el } \\
\text { esplendor que el favor real daba } \\
\text { a su casa. } \\
\text { [Sesplugues. La Fayette, I924:24] }\end{array}$ \\
\hline & $\begin{array}{c}\text { TABLA IV. } \\
\text { ESTRUCTURAS SINTÁCTICAS }\end{array}$ & \\
\hline FRANCÉS & CATALÁN & ESPAÑOL \\
\hline $\begin{array}{l}\text { Je suis si haïe de la reine et de la } \\
\text { duchesse de Valentinois... } \\
\qquad \text { [La Fayette 2000: Io] } \\
\text { j’ai envoyé une de mes femmes lui } \\
\text { faire des excuses. } \\
\text { [La Fayette, 2000: 64] }\end{array}$ & $\begin{array}{l}\text { Sóc tan odiada per la reina i per } \\
\text { la duquessa... } \\
\text { [Marquina. La Fayette I923:20] } \\
\text { he enviat una de les meves cam- } \\
\text { breres perquè li presentés les } \\
\text { meves excuses. } \\
\text { [Marquina. La Fayette, } \\
\text { I923:I46 }\end{array}$ & $\begin{array}{l}\text { Me odian tanto la reina y la } \\
\text { duquesa de Valentinois... } \\
\quad \text { Sesplugues. La Fayette I924:30] } \\
\text { y he enviado a una de mis donce- } \\
\text { llas para presentarle mis excusas. } \\
\text { [Sesplugues. La Fayette, I924:I9I] }\end{array}$ \\
\hline
\end{tabular}


elabora además partiendo de una edición francesa distinta de la que parte la versión catalana. Lo puede atestiguar la distribución del texto en párrafos: a menudo es distinta en las dos traducciones, así como también es distinto el sistema de representación del discurso directo (no siempre hay coincidencias en el uso de comillas o guiones). Un sistema distinto en el uso de los signos de puntuación o de algunas grafías para representar antropónimos germánicos acaba confirmando la independencia de las dos traducciones. Finalmente, como rasgo curioso de la versión de Sesplugues, cabe señalar su interés por facilitar al lector el seguimiento del hilo narrativo de la historia repitiendo o explicitando de forma completa el nombre y tratamiento dispensado a los personajes (recurso más cercano al original francés):

TABLA V.

CATEGORÍAS GRAMATICALES Y EL USO DE LOS TIEMPOS VERBALES

\begin{tabular}{|c|c|c|}
\hline FRANCÉS & CATALÁN & ESPAÑOL \\
\hline étant bien aise de continuer & segura de continuar fàcilment & era muy fácil continuar \\
\hline [La Fayette, 2000: 30] & [M. La Fayette, I923:69] & [S. La Fayette, I924:87] \\
\hline Le chevalier de Guise, qui & El cavaller de Guisa, que li tenia & El caballero de Guisa, que conti- \\
\hline l'adorait toujours... & adoració... & nuaba adorándola... \\
\hline [La Fayette, 200o: I4] & [M. La Fayette, I923:33] & [S. La Fayette, I924:40] \\
\hline -Est-il possible, lui disait-il, que... & És possible — digué- que...? & ¿Es posible - le decía- que... \\
\hline [La Fayette, 2000: II] & [M. La Fayette, I923:28] & [S. La Fayette, 1924:34] \\
\hline \multirow{2}{*}{$\begin{array}{l}\text { qui avait toujours demeuré chez } \\
\text { elle }\end{array}$} & havia romàs a casa seva & continuaba sin salir de casa \\
\hline & [M. La Fayette, I923:I43] & [S. La Fayette, I924:187] \\
\hline [La Fayette, 2000: 63] & & \\
\hline
\end{tabular}

TABLA VI.

FORMAS LÉXICAS

FRANCÉS

Toutes ces différentes cabales avaient de l'émulation et de l'envie les unes contre les autres.

[La Fayette, 2000: 9]

Le cardinal de Wolsey [...] avait prétendu au pontificat; et mal satisfait de l'Em-pereur, qui ne l'avait pas soutenu dans cette prétention, il résolut de s'en venger, et d'unir le roi, son maître, à la France.
CATALÁN

Totes aquestes tertúlies intrigants tenien enveja unes d'altres.

[M. La Fayette, I923:2I]

El cardenal Wolsey [...] havia pretès el pontificat, $i$, descontent de l'emperador, que no l'havia ajudat en aquesta pretensió, prengué determini de venjar-se'n aliant el rei, son senyor, amb la França.

[M. La F ayette, I923:76]
ESPAÑOL

Todas estas diferentes agrupaciones sentían emulación y envidia las unas contra las otras.

[S. La Fayette, I924:26]

El cardel Volsey [...] aspiraba al pontificado, y disgustado con el emperador porque no le había apoyado en su pretensión, resolvió vengarse uniendo a su rey y señor con Francia.

[S. La Fayette, 1924:97] 
Lo que sí podemos afirmar con rotundidad es que la primera edición de La princesa de Clèves publicada después de la Guerra Civil, en 52 I94I, por Editorial Apolo, de Barcelona, en su Biblioteca Freya, cuya traducción lleva la firma de F. S., es un auténtico calco o plagio de la de J. Sesplugues, con algún pequeño retoque disimulador. Editorial Apolo constituye, en palabras de Llanas (2005: 313), un paradigma de los enormes déficits de información que acumula la historia de la edición. En efecto, tratándose de una editorial contemporánea, resultan hoy difíciles las tentativas para dilucidar su origen y trayectoria. Nacida, al parecer, a finales de los años 20, propiedad de Emili Pascual, vive su esplendor durante la Segunda República, etapa en la que da a conocer sus títulos más relevantes. Hacia 1938 Apolo disponía de seis colecciones, orientadas principalmente a las ciencias sociales (psicología, antropología, sociología, filosofía, biografías, etc.) y, sobre todo, a la literatura en prosa. Además de la colección Grandes Novelas, que incorpora Thomas Mann, Arthur Schnitzier o Giovanni Papini, Apolo publica la Biblioteca Freya, de narrativa corta, entre cuyos autores figuran, en la etapa republicana, Stefan Zweig, Aldous Huxley o Lajos Zilahy. Se trata, como apunta uno de les primeros catálogos de la editorial, de «satisfacer los nobilísimos anhelos del público que lee» y de «incorporar al acervo literario [...] todos aquellos autores de universal prestigio y cuyas obras [...] han de merecer la más excelente acogida». (Llanas, 2005: 315). Apolo terminará siendo absorbida en I95I por el conglomerado editorial de Josep Janés.

Según Holzbache, autora de la última traducción al español (La Fayette, I987), las iniciales F. S. de la traducción de Apolo pertenecerían a Federico Carlos Sáinz de Robles y Correa (Madrid, I898-1983), escritor, dramaturgo, historiador, lexicógrafo, crítico literario, historiador de la literatura, folclorista, bibliógrafo y ensa-

TABLA VII.

FORMAS COMPLETAS DE NOMBRE Y TRATAMIENTO

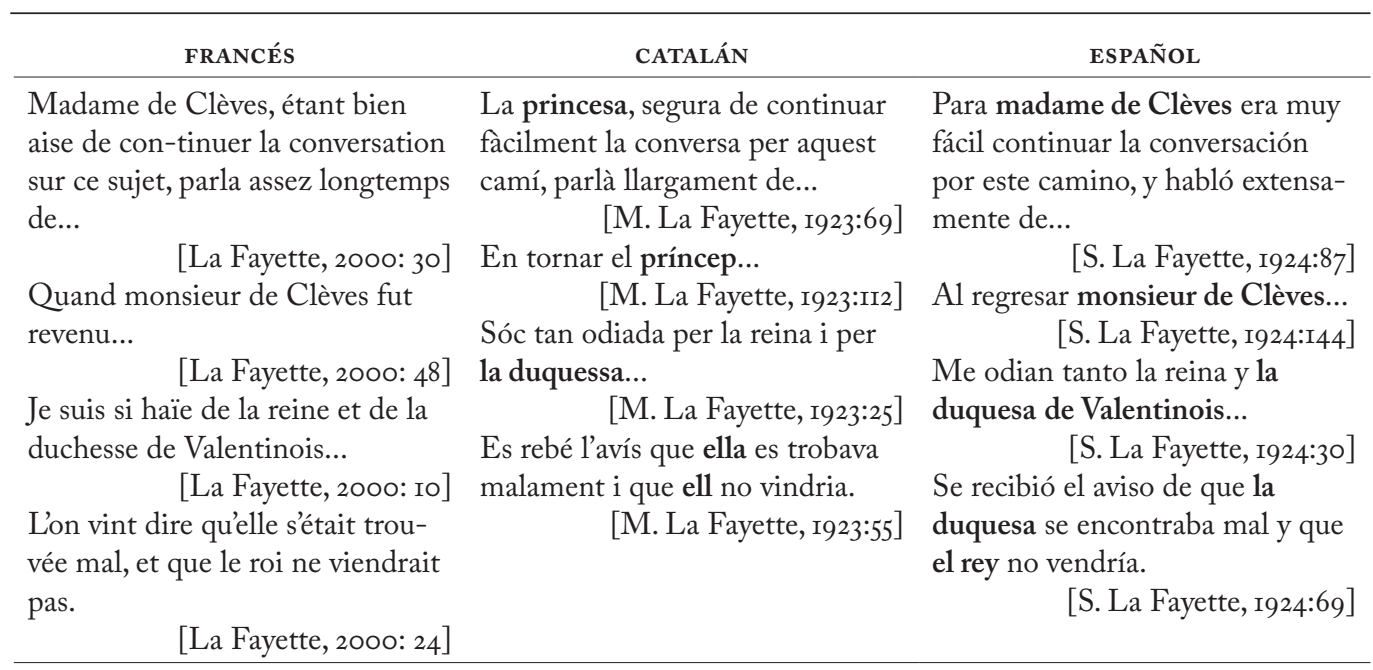


yista. Se puede juzgar dicho plagio cotejando distintos fragmentos de la obra (tablas 8 y 9). Puede observarse, además, en los fragmentos de la traducción de 1987 su independencia con respecto a la traducción de i924:

\section{CONCLUSIONES}

A modo de conclusión se puede afirmar que las dos primeras traducciones peninsulares de La Princesse de Clèves (1678) llegan con notable retraso: unos 245 años. No obstante, lo que suscita la génesis de las versiones al catalán y al español de la novela son en ambos casos factores de política cultural. El texto clásico del XVII de Madame de La Fayette es precursor de la novela moderna francesa al tiempo que intensifica la presencia de la mujer en la literatura de la época, ya sea como autora y como protagonista de una historia.

La traducción catalana de Marquina, de 1923, es sin duda el resultado de la política lingüística y literaria emprendida por el Noucentisme, en la etapa de la Mancomunitat de Catalunya, en su afán de favorecer las traducciones como procedimiento para obtener un catalán literario común, moderno y flexible, especialmente para usos elevados, y como superación del localismo

TABLA VIII.

PARANGÓN ENTRE EL TEXTO DE I 924 Y DE I 94 I

FRANCÉS

Vous savez l'amitié qu'il y a entre Sancerre et moi ; néanmoins il devint amoureux de madame de Tournon, il y a environ deux ans, et me le cacha avec beaucoup de soin, aussi bien qu'à tout le reste du monde. J'étais bien éloigné de le soupçonner. Madame de Tournon paraissait encore inconsolable de la mort de son mari, et vivait dans une retraite austère. La soeur de Sancerre était quasi la seule personne qu'elle vit, et c'était chez elle qu'il en était devenu amoureux.

[La Fayette, 2000: 24]

SESPLUGUES. LA FAYETTE, I924

Ya sabéis la amistad que hay entre Sancerre y yo. No obstante, hará unos dos años que se enamoró de madame de Tournon, y me lo ocultó con el mismo sigilo que a los demás. Estaba yo muy lejos de sospecharlo. Madame de Tournon, que parecía aún inconsolable por la muerte de su esposo, vivía en un austero retiro. Casi la única persona que veía era la hermana de Sancerre, y en su misma casa fué donde éste se enamoró de ella.
F. S. LA FAYETTE, I94I Ya sabéis la amistad que hay entre Sancerre y yo. No obstante, hará unos dos años que se enamoró de la señora de Tournon, y me lo ocultó con el mismo sigilo que a los demás. Estaba yo muy lejos de sospecharlo. La señora de Tournon, que parecía aún inconsolable por la muerte de su esposo, vivía en un austero retiro. La hermana de Sancerre era casi la única persona que veía, y fué en su misma casa donde éste se enamoró de ella.

HOLZBACHER. LA FAYETTE, I 987

Sabéis la amistad que existe entre Sancerre y yo, sin embargo, se enamoró de la señora de Tournon hace unos dos años, y me lo ocultó con sumo cuidado, así como a todo el mundo. Yo estaba muy lejos de sospecharlo. La señora de Tournon parecía inconsolable por la muerte de su marido y vivía en un retiro austero. La hermana de Sancerre era casi la única persona a quien veía, y fue en su casa donde éste se enamoró de ella.

[p. I4I]

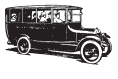


TABLA IX.

PARANGÓN ENTRE EL TEXTO DE I 924 Y DE I 94 I

\section{FRANCÉS}

-Et monsieur de Nemours, lui dit-il, ne l'avez-vous point vu, ou l'avez-vous oublié ?

-Je ne l'ai point vu, en effet, répondit-elle; je me trouvais mal, et j’ai envoyé une de mes femmes lui faire des excuses.

[La Fayette, 2000: 64]

\begin{tabular}{l} 
SESPLUgues. LA FAYETTE, I924 \\
\hline - ¿Y a monsieur de Nemours? ¿Es \\
que no le habéis visto o es que le \\
habéis olvidado? \\
-No le he visto, en efecto -res- \\
pondió ella-. Me encontraba \\
indispuesta y he enviado a una \\
de mis doncellas para presentarle \\
mis excusas.
\end{tabular}

[p. I90-I9I] mis excusas.
- ¿Y al señor de Nemours? ¿Es que no le habéis visto o es que le habéis olvidado? -No le he visto, en efecto -respondió ella-. Me sentía indispuesta y he enviado a una de mis doncellas para presentarle mis excusas.

HOLZBACHER. LA FAYETTE, I987

- ¿Y el señor de Nemours, no lo

habéis visto, o lo habéis olvidado?

- No lo he visto, en efecto -respondió ella-, me encontraba mal $\mathrm{y}$ he enviado a una de mis doncellas a pedirle que me disculpase.

[p. 237]
[p. I09]

dando a conocer los principales textos de la literatura universal.

En el caso de la primera versión al español, de I924, por J. Sesplugues (pseudónimo), cabe decir que muy probablemente fue posible gracias al interés por crear, por primera vez en la historia de la edición hispana, una industria editorial Calpe, El Sol, La Papelera Españolaque pudiera difundir una corriente ideológica laicista, progresista y renovadora, al tiempo que ofreciese, en el ámbito literario, una selección universal del pensamiento y de la estética a través de las obras clásicas más preciadas, en un formato y a unos precios asequibles al gran público. Y la traducción del desconocido Sesplugues, a la que siguieron otras, lo atestigua. Será necesario no desfallecer en el intento de descubrir la identidad del responsable civil de la primera traducción al español de esta joya del XVII francés.
RECIBIDO EN OCTUBRE DE 2014

ACEPTADO EN FEBRERO DE 2015

VERSIÓN FINAL DE SEPTIEMBRE DE 2015

\section{REFERENCIAS}

Bacardí, Montserrat (2007). «La traducció del català al castellà: una tradició aleatòria» [en línea]. Revista d'bistòria de la traducció. <www.traducionliteraria.org/I6II/ art/ bacardi.htm $>$ [Consulta: I2 diciembre 20I2.]

Blottier, Henry Pierre, Catherine Vandel-Isaakidis (I99I). La Princesse de Clèves, París: Bordas.

Cabrera, Mercedes (1994). La industria, la prensa y la política. Nicolás María Urgoiti (I869-195I), Madrid: Alianza Editorial.

Chaurand, Jacques (I999). Nouvelle histoire de la langue française, París: Seuil.

Cherbuliex, Victor (I9II). L'idéal romanesque en France de I6ro à I8I6, París: Librairie Hachette.

Coromina Pou, Eusebi (2012). «Dues traduccions catalanes de la Princesse de Clèves de Madame de La Fayette. Un clàssic del XVII que marca l'accés de la dona a la novel-la». Quaderns. Revista de Traducció, 19, pp. 155-167. 
«Editorial» (I9I7), El Sol, I.XII.r9I7, p. I.

Ginavel, Joan (1934). "Cinc-cents pseudònims catalans». Butlletí de la Biblioteca de Catalunya, 8, pp. I2I-I42.

La Fayette, Madame de (I923). La princesa de Clèves. Trad. de Rafael Marquina. Barcelona: Editorial Catalana.

- (I924). La princesa de Clèves. Trad. de J. Sesplugues. Madrid: Calpe.

- (I94I). La Princesa de Clèves. Trad. de F. S. [Federico Carlos Sáinz de Robles, padre]. Barcelona: Apolo.

- (1987). La Princesa de Clèves. Trad. de Ana María Holzbacher. Madrid: Cátedra.

- (2000). La Princesse de Clèves. Éditions eBooksFrance [en línea]. <http://www.ebooksgratuits. com/ebooksfrance/madame_de_lafayette_princesse_de_cleves.pdf $>$ [Consulta: 20 diciembre 20I3.]

Lathuillère, Roger (1966). La Preciosité. Étude historique et linguistique, Ginebra: Droz.

Lever, Maurice (I98I). Le roman français au XVIIe siècle, París: PUF.

Llanas, Manuel (2005). L'edició a Catalunya: el segle XX (fins a I939), Barcelona: Gremi d'Editors de Catalunya.

Llanas, Manuel, Ramon Pinyol (200I). «Prosistas y poetas franceses (hasta el siglo XVIII) traducidos al catalán en el siglo XX», en F. Lafarga, Antonio Domínguez (eds.), Los clásicos franceses en la España del siglo XX. Estudios de traducción y recepción. Barcelona: Promociones y Publicaciones Universitarias, S.A., pp. 23-37.
Malandain, Pierrre (1989). Madame de Lafayette. La Princesse de Clèves, París: Puf.

Malé, Jordi (2007). "Una llengua en plena ebullició”. Els traductors davant el català literari a les primeres dècades del segle XX». Quaderns. Revista de Traducció I4, pp. 79-94.

Pericay, Xavier, Ferran Toutain (1996). El malentès del noucentisme. Traducció i plagi a la prosa catalana moderna, Barcelona: Proa.

Pingaud, Bernard (1978). Mme de La Fayette, París: Seuil.

Pommier, René (I990). Études sur «La Princesse de Clèves», París: Eurédit.

Rapin, René (I677). Instructions pour l'bistoire, París: S. Mabre - Cramoisy.

Sánchez Vigil, Juan Miguel (2005). Calpe. Paradigma editorial (I9I8-I925), Gijón: Trea.

- (2006). «La editorial CALPE y el Catálogo General de I923». Documentación de las Ciencias de la Información 28, pp. 259-277.

Serra i Casals, Enric (I996). "La revista "Teatralia" com a plataforma noucentista». Els Marges 55, pp. 7-28.

Sesplugues, J. (1926). "Verdaguer i la seva poesia», Segarra I5-VII-I926, pp. I7-I8.

Sgard, Jean (2000). Le Roman français à l'âge classique, París: Librairie Générale Française.

Timmermans, Linda (I993). L'Accès des femmes à la culture (1598-1715). Un débat d'idées de saint François de Sales à la marquise de Lambert, París: Honoré Champion.

«Un extraordinari de "Segarra" (I926), La Veu de Catalunya I7-VII-I926, p. 9 .

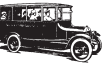

55 